\title{
Contrast-enhanced harmonic endoscopic ultrasound-guided ethanol injection for a small hepatocellular carcinoma
}

Locoregional ablation represents the mainstay approach for early hepatocellular carcinoma (HCC). Percutaneous treatments are performed under ultrasound guidance to select a safe window to reach the nodule with a dedicated device [1].

We present a 76-year-old female patient, with hepatitis C-related cirrhosis, whose history included a wedge resection for a segment-6 HCC and subsequent transarterial chemoembolization. In 2014, computed tomography showed a $14-\mathrm{mm}$ nodule with typical hallmarks for HCC located in the deep subcapsular portion of hepatic segment 2.

Further surgery was contraindicated and percutaneous ablation was not feasible because of left portal vein interposition along the theoretical needle path. After multidisciplinary discussion, endoscopic ultrasound (EUS)-guided ethanol injection was attempted. A GF-UCT180 echoendoscope (Olympus, Japan) was used in conjunction with a Prosound-Alpha-10 system (Aloka, Japan). In order to adequately identify the tumor extent, contrast-enhanced harmonic EUS (CH-EUS) was used after Sonovue injection (Bracco International, Netherlands) ( Fig. 1, \Video 1) [2]. A 22-gauge needle (Expect SlimLine; Boston-Scientific, USA) was inserted in the deepest portion of the nodule and $10 \mathrm{~mL}$ of ethanol were injected ( $\mathbf{F i g . 2}$ ). The procedure was well tolerated and no adverse events occurred.

Contrast-enhanced ultrasound 40 days later showed suspected persistence of the HCC. CH-EUS showed a mainly nonenhanced area in the region that had been previously treated, which was compatible with necrosis. However, in its peripheral portion, a tiny $(3-\mathrm{mm})$ residual arterial vascularization was confirmed ( $\mathbf{F i g . 3}$ ). In the same session, the patient underwent an uneventful second ethanol injection $(3 \mathrm{~mL})$ under $\mathrm{CH}$-EUS guidance ( $\vee$ Fig. 4, $>$ Video 1 ).

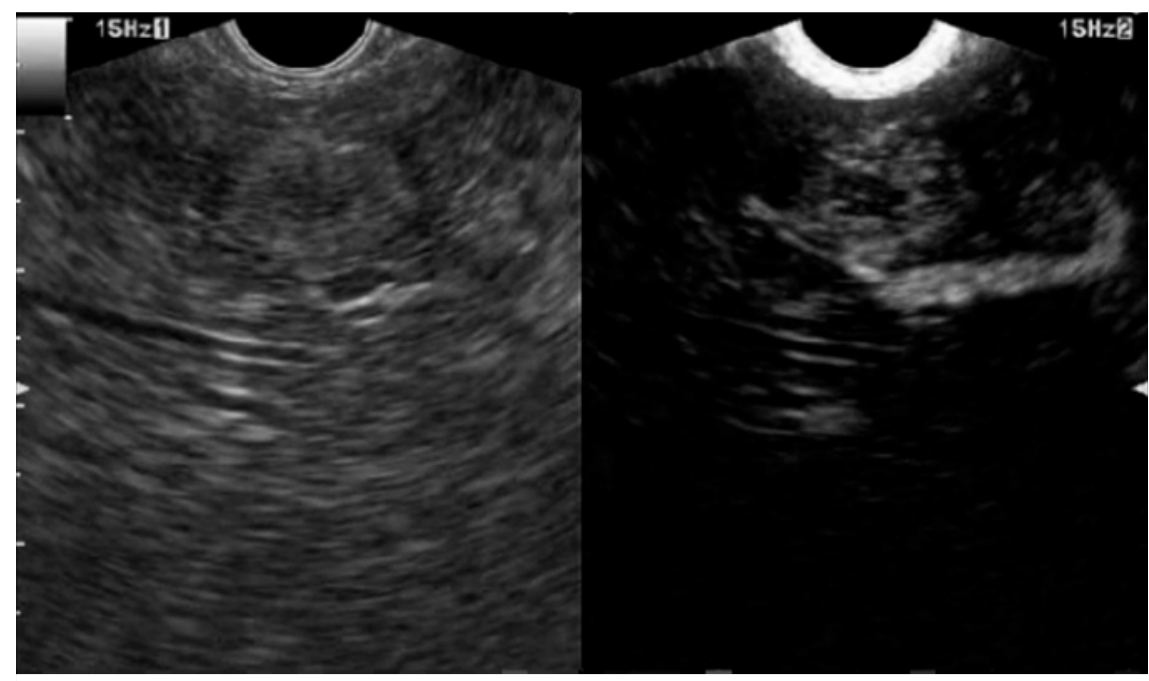

- Fig. 1 Contrast-enhanced harmonic endoscopic ultrasound (CH-EUS) evaluation of a 14-mm hepatocellular carcinoma (HCC) in segment 2. Time: 20 seconds after contrast injection.

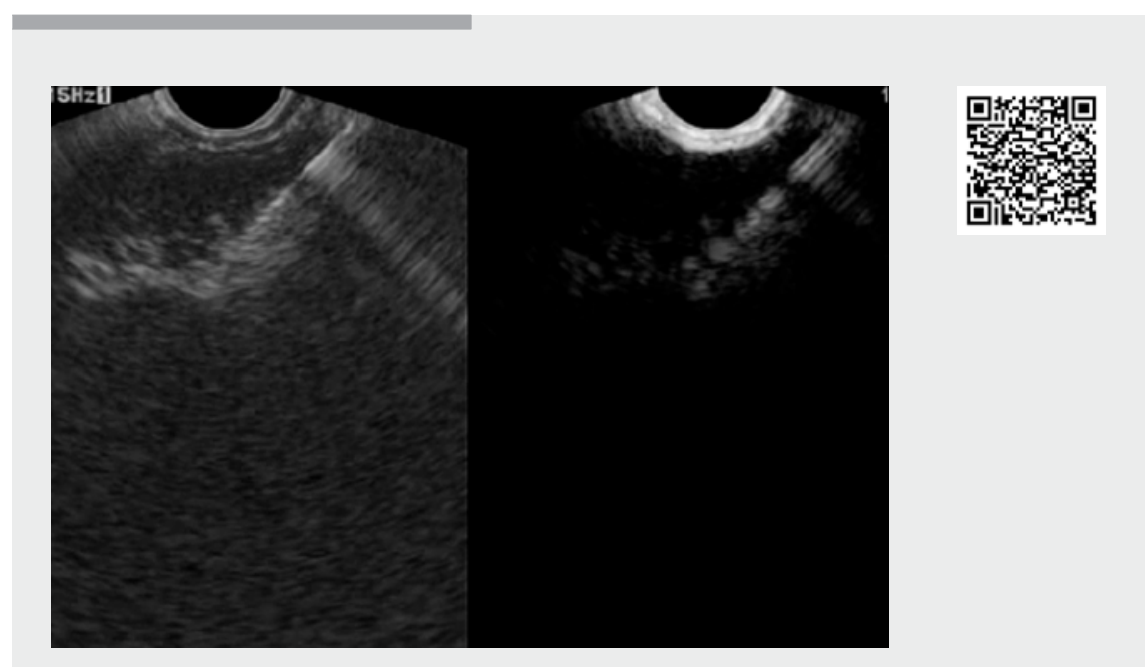

$\checkmark$ Video 1 Small hepatocellular carcinoma treated by contrast-enhanced harmonic endoscopic ultrasound-guided (CH-EUS) ethanol injection.

Follow-up at 3, 6 and 12 months with computed tomography showed neither local nor distant recurrence. The patient continued follow-up for a further 8 months until death from causes unrelated to her liver.

This case suggests that EUS-guided ablation of HCC represents a valid alternative to percutaneous ablation when the latter is not feasible or is contraindicated. Interestingly, $\mathrm{CH}$-EUS may help to identify viable areas of HCC and allow targeted treatment under direct dynamic visualization of tumor vessels [3-5].

Endoscopy_UCTN_Code_TTT_1AS_2AG 


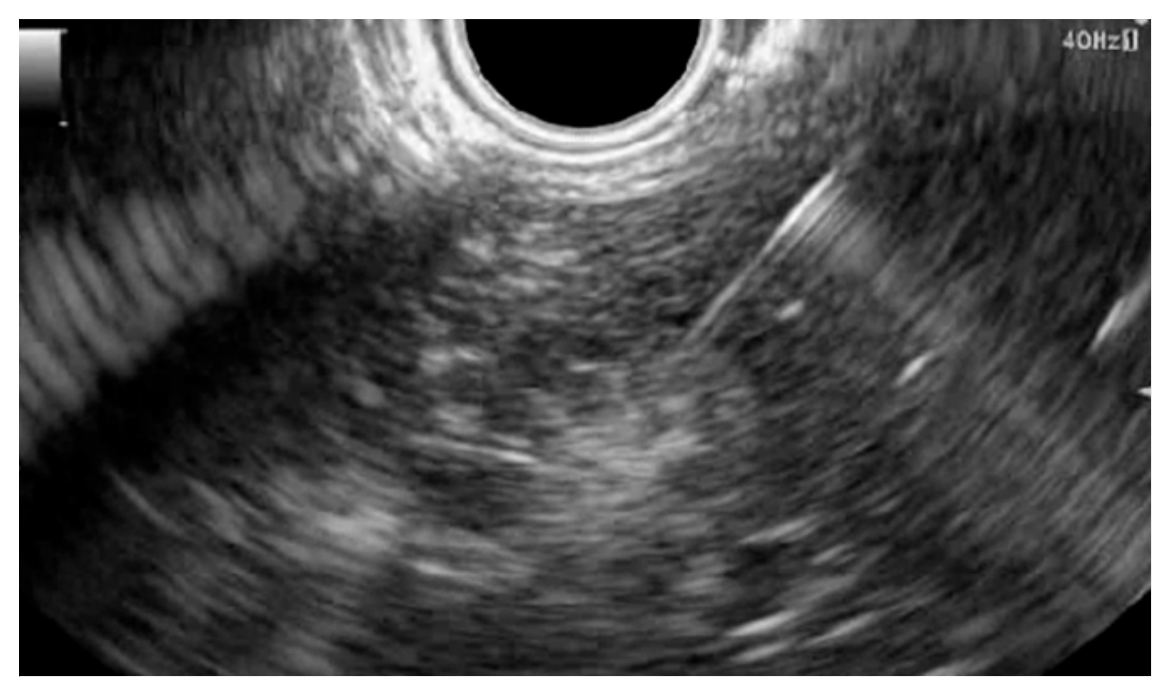

- Fig. 2 EUS-guided ethanol ablation of the 14-mm hepatic segment-2 HCC.

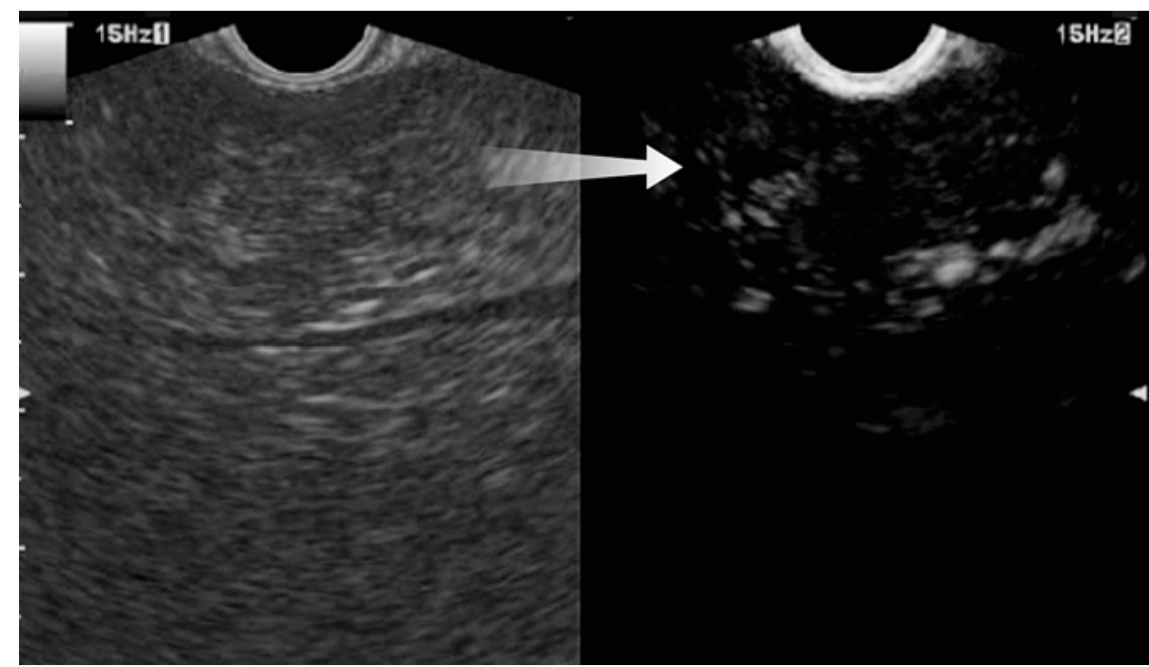

Fig. 3 Identification of a small (2-3-mm) residual HCC on CH-EUS. Time: 27 seconds after contrast injection.

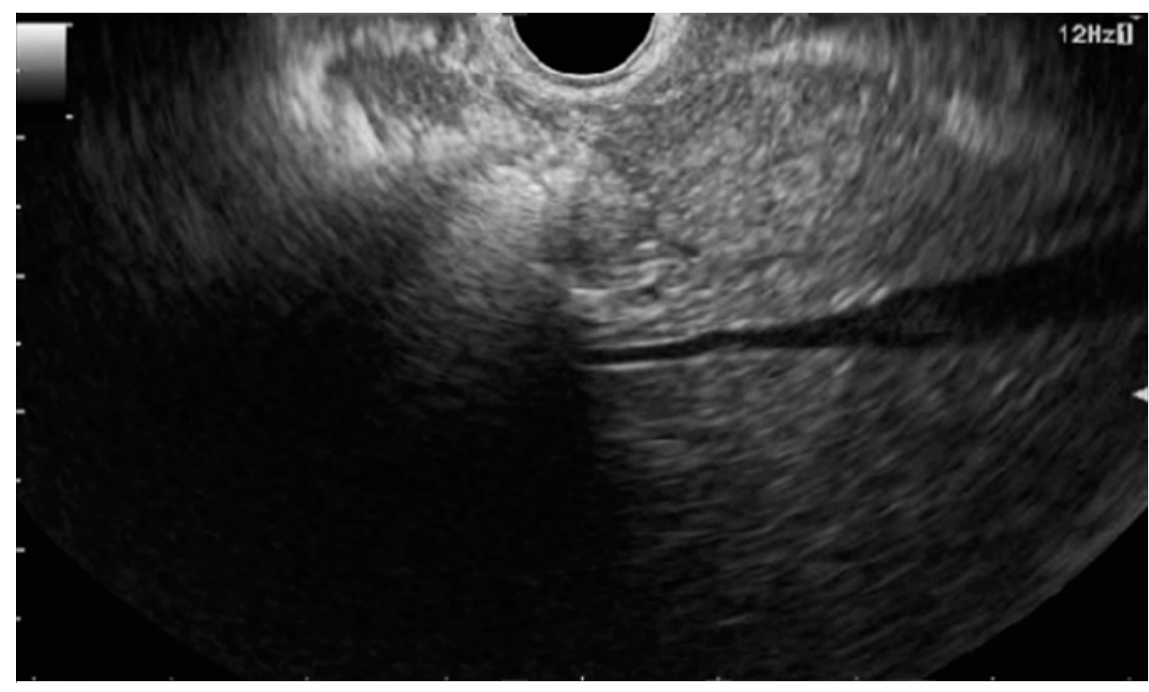

D Fig.4 EUS evaluation after second ethanol injection into the residual HCC.
Competing interests

None

The authors

\section{Andrea Lisotti ${ }^{1}$, Fabio Piscaglia ${ }^{2}$, Pietro Fusaroli ${ }^{1}$}

1 Gastroenterology Unit, Department of Medical and Surgical Sciences, University of Bologna/Hospital of Imola, Italy

2 Internal Medicine Unit, Department of Medical and Surgical Sciences, University of Bologna, S.Orsola-Malpighi Hospital, Bologna, Italy

\section{Corresponding author}

\section{Pietro Fusaroli, MD}

Gastroenterology Unit, Hospital of Imola, Via Montericco 4, 40026 Imola (BO), Italy Fax: +39-0542-662409 pietro.fusaroli@unibo.it

\section{References}

[1] Dietrich CF, Lorentzen T, Appelbaum L et al. EFSUMB guidelines on interventional ultrasound (INVUS), Part III - Abdominal treatment procedures (long version). Ultraschall Med 2016; 37: E1-E32

[2] Lisotti A, Serrani M, Caletti G et al. EUS liver assessment using contrast agents and elastography. Endosc Ultrasound 2018; 7: 252 256

[3] Fusaroli P, Napoleon B, Gincul R et al. The clinical impact of ultrasound contrast agents in EUS: a systematic review according to the levels of evidence. Gastrointest Endosc 2016; 84: 587-596

[4] Fabbri C, Luigiano C, Lisotti A et al. Endoscopic ultrasound-guided treatments: are we getting evidence based - a systematic review. World J Gastroenterol 2014; 20 : $8424-8448$

[5] Fusaroli P, Jenssen C, Hocke M et al. EFSUMB guidelines on interventional ultrasound (INVUS), Part V - EUS-guided therapeutic interventions (short version). Ultraschall Med 2016; 37: 412-420

\section{Bibliography}

DOI https://doi.org/10.1055/a-0915-1385

Published online: 4.6.2019

Endoscopy 2019; 51: E317-E318

(c) Georg Thieme Verlag KG

Stuttgart · New York

ISSN 0013-726X 